\title{
An Unusual Case of Cervical Cancer with Inguinal Lymph Node Metastasis: A Case Report and Review of the Literature
}

\author{
Guler Yavas ${ }^{*}$, Ozlem Ata $^{2}$, Cetin Celik ${ }^{3}$ \\ ${ }^{1}$ Selcuk University, Department of Radiation Oncology, Konya, Turkey \\ ${ }^{2}$ Selcuk University, Department of Medical Oncology, Konya, Turkey \\ ${ }^{3}$ Selcuk University, Department of Obstetrics and Gynecology, Division of Gynecologic Oncology, Konya, Turkey
}

"Corresponding Author: Guler Yavas, Selcuk University Faculty of Medicine, Department of Radiation Oncology Konya, Turkey, Selcuklu, Konya, Turkey, Tel: +90-332-224-40-85; Fax: +90-332-2417791; E-mail: guler.aydinyavas@gmail.com

Received: 12 July 2017; Accepted: 17 July 2017; Published: 21 July 2017

\begin{abstract}
There is limited data regarding to treatment of cervical cancer patients with isolated non-regional lymph node metastasis. Herein we report a case of cervical cancer with left inguinal lymph node metastasis at the time of diagnosis. A 52-year-old woman referred to gynecologic oncology department with postmenopausal vaginal bleeding history for a year. Gynecological examination revealed a bulky cervical tumor with left parametrial invasion. The biopsy of this lesion revealed a squamous cell carcinoma of the cervix. A pelvic magnetic resonance imaging (MRI) demonstrated a bulky cervical mass with a parametrial extension on left side, in addition to the suspicious lymph nodes in the para-aortic, pelvic and left inguinal chains. The patient was underwent extraperitoneal para-aortic lymph node dissection and left inguinal lymphadenectomy. Histopathological examination of the lymph nodes revealed that there were metastatic lymph nodes in the para-ortic, and left inguinal chains. We planned neoadjuvant chemotherapy consisting of paclitaxel and carboplatin; and we decided to plan adjuvant treatment with respect to the treatment response to the induction chemotherapy. The control computed tomography after 6 cycle of chemotherapy showed that there was a good-partial response; therefore definitive radiotherapy was planned. A month after the radiotherapy pelvic MRI was performed and it showed a complete response. After 38 months of follow-up the patient admitted to the emergency department with ileus. The patient developed sepsis and forty months after the completion of the radiotherapy the patient expired because of septic shock.
\end{abstract}


Keywords: Cervical cancer; Inguinal lymph node; Neoadjuvant chemotherapy; Radiotherapy

\section{Introduction}

Cervical cancer is the third most common gynecologic cancer diagnosis and cause of death among gynecologic cancers in the United States [1]. It is clinically classified according to the International Federation of Gynecology and Obstetrics (FIGO) clinical staging system [2]. Although the system does not include evaluation of lymph node involvement, lymph node status remains the single most important prognostic factor and major determinant of treatment modality in patients with the diagnosis of cervical cancer [3-5].

Cervical cancer lymphatic drainage can be divided into three levels: The level I lymph nodes consist of parametrial and obturator lymph nodes. Internal and external iliac lymph nodes can be considered as level II lymph nodes, and the common iliac and inguinal lymph nodes as level III nodes. [6]. The predicted lymphatic drainage pattern in cervical cancer patients is stepwise progression from the cervical stroma and serosal lymphatics to the nodal groups in the parametrium and pelvis, although there may be significant variations. The most important route runs along the lateral parametrium to the obturator, external and internal iliac and common iliac lymph nodes. There are many studies in the literature including those utilizing the sentinel lymph node mapping technique, emphasize that any of the pelvic lymph node groups, and even paraaortic lymph nodes, may contain the first draining lymph node and may be the first site of nodal metastasis [7, 8]. In a study Ouldamer and colleagues found that $83.7 \%$ of detected sentinel lymph nodes in patients with cervical cancer were in expected localizations (i.e., external iliac, obturator, internal iliac or interiliac). The unusual localizations were: $6.6 \%$ in the common iliac chain, $4.31 \%$ parametrial, $1.26 \%$ sacral, $2 \%$ in the lower para-aortic area and $0.07 \%$ in the inguinal chain [9]. Therefore inguinal lymph node metastasis is rarely seen in cervical cancer patients. According to the TNM classification of malignant tumors (TNM) inguinal lymph nodes are considered as non-regional lymphatics in cervical cancer therefore, involvement in an inguinal lymph node is accepted as distant metastasis (M1) [10].

There is limited data regarding about the treatment of cervical cancer patients with isolated non-regional lymph node metastasis. Therefore the treatment of cervical cancer patients with inguinal lymph node metastasis is still challenging. Herein, we report a case of cervical cancer who had an inguinal lymph node metastasis at the time of diagnosis.

\section{Case Presentation}

A 52-year-old woman referred to gynecologic oncology department of our university with postmenopausal vaginal bleeding history for a year. Her previous history was uneventful. Gynecological examination revealed an approximately $6.5 \mathrm{~cm}$ bulky cervical tumor with left parametrial invasion and extension to the upper part of the vagina. The biopsy of this lesion revealed a squamous cell carcinoma of the cervix. The flexible cystourethroscopy revealed a $5 \mathrm{~cm}$ mass beginning from the base of the bladder and extending through the posterior wall of the bladder on the left side. There was no observable mass in the bladder. Rectoscopy was completely normal. A pelvic magnetic resonance imaging (MRI) demonstrated a $7 \times 6 \mathrm{~cm}$ cervical mass arising from the cervix with a parametrial extension on the left side. There were suspicious lymph nodes in the left parailiac chain $(2 \times 1.5 \mathrm{~cm})$ and right parailiac chain $(2.2 \times 1.4 \mathrm{~cm})$; and left inguinal chain $(2.2 \times 1.7 \mathrm{~cm})$ (Figure 1). At the time of diagnosis the Ca-125 level was $46.09 \mathrm{U} / \mathrm{mL}$ (normal range: 0-35 U/mL). The patient was underwent extraperitoneal paraaortic lymph 
node dissection and left inguinal lymphadenectomy. Histopathological examination of the lymph nodes revealed a metastatic paraaortic lymph node (1/24) in addition to a metastatic left inguinal lymph node (1/3). There was no any other distant metastasis. The patient was staged as IVB according to FIGO classification system. We planned neoadjuvant chemotherapy consisting of paclitaxel $(290 \mathrm{mg}$ ) and carboplatin (700 mg); and we decided to plan adjuvant treatment with respect to the treatment response to the induction chemotherapy. After 2 cycles of chemotherapy, we performed computed tomography (CT) in order to define the treatment response. The cervical mass was shrunk and the bladder invasion was completely inapparent. There was only grade-1 neutotoxicity during the first 2 cycle of chemotherapy. After $4^{\text {th }}$ cycle the patient developed grad-4 neutropenia; however primary granulocyte- colony-stimulating factor (G-CSF) prophylaxis was successful; therefore we could complete 6 cycles of neoadjuvant chemotherapy. The Ca-125 level was $22.33 \mathrm{U} / \mathrm{mL}$ (normal range: $0-35 \mathrm{U} / \mathrm{mL}$ ) after 4 cycles of chemotherapy.

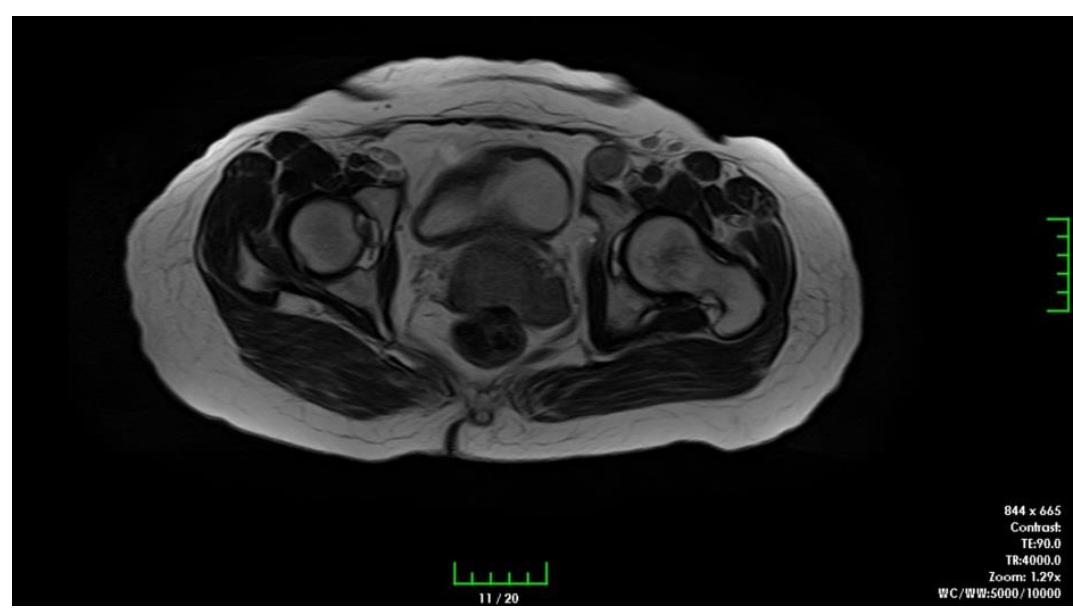

(a)

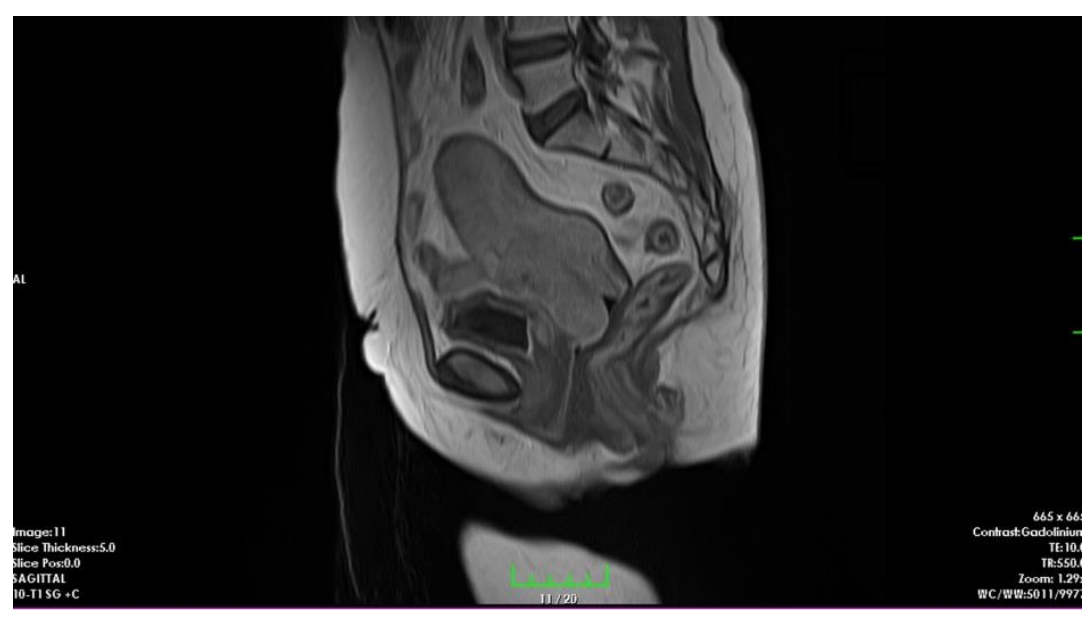

(b)

Figure 1: Pelvic MRI demonstrated a cervical mass with a parametrial extension on the left side, suspicious lymph nodes in the left parailiac and right parailiac chain); and left inguinal chain, (a) axial view, (b) sagittal view.

The control CT after the 6 cycle of chemotherapy showed that there was a good-partial response to the neoadjuvant chemotherapy; therefore we planned definitive radiotherapy (Figure 2). We performed external pelvic+paraaortic + 
bilateral inguinal irradiation using 3-dimensional conformal radiotherapy to a dose of 45 Gy in 25 daily fractions of $1.8 \mathrm{~Gy}$ each. And we used midline blocks up to $50.4 \mathrm{~Gy}$ to the pelvic and left inguinal lymph nodes. Treatment was given without interruption. She had only grade 1 cystitis and diarrhea which respond to medical treatments. After $28^{\text {th }}$ fraction of external radiotherapy the patient underwent cervical brachytherapy to a dose of 28 Gy in 4 fractions over 2 weeks.

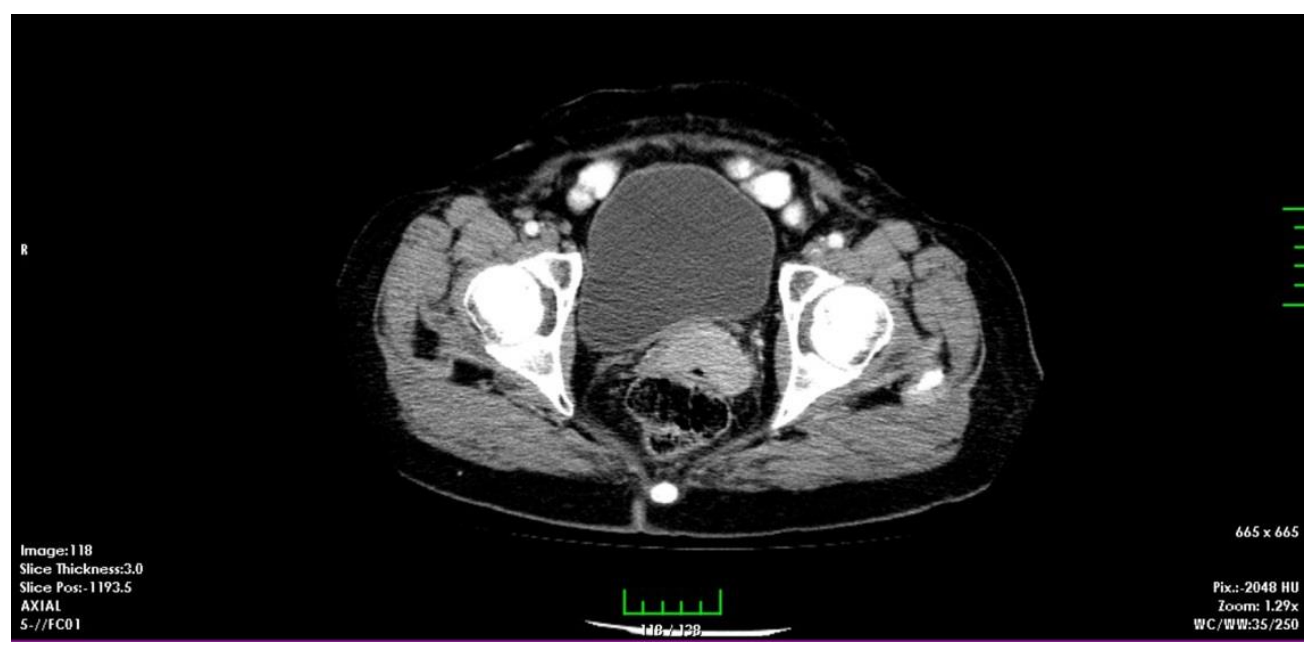

Figure 2: The CT images after 6 cycles of neoadjuvant chemotherapy showing a good-partial response.

One month after the radiotherapy we performed pelvic MRI which showed us a complete response to the treatment (Figure 3). The patient went on routine follow-up. After 38 months of follow-up the patient admitted to the emergency department with ileus. The patient developed sepsis and forty months after the completion of the radiotherapy the patient expired because of septic shock.

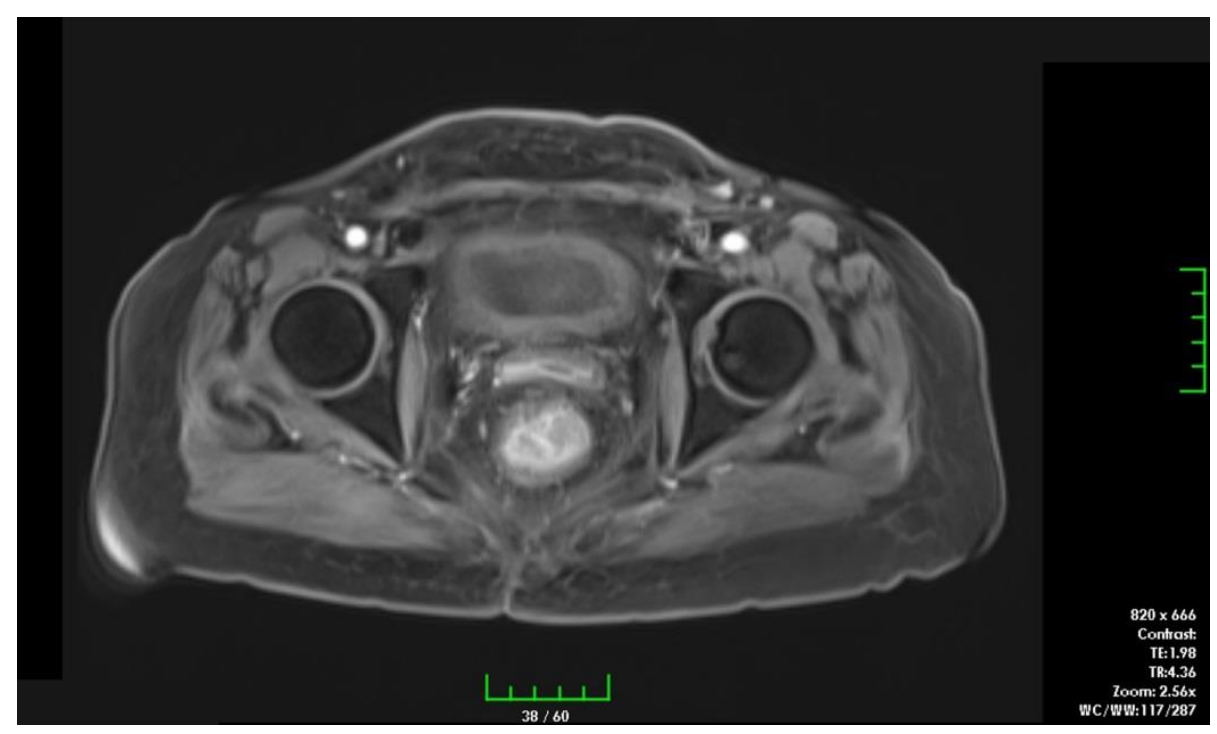

(a) 


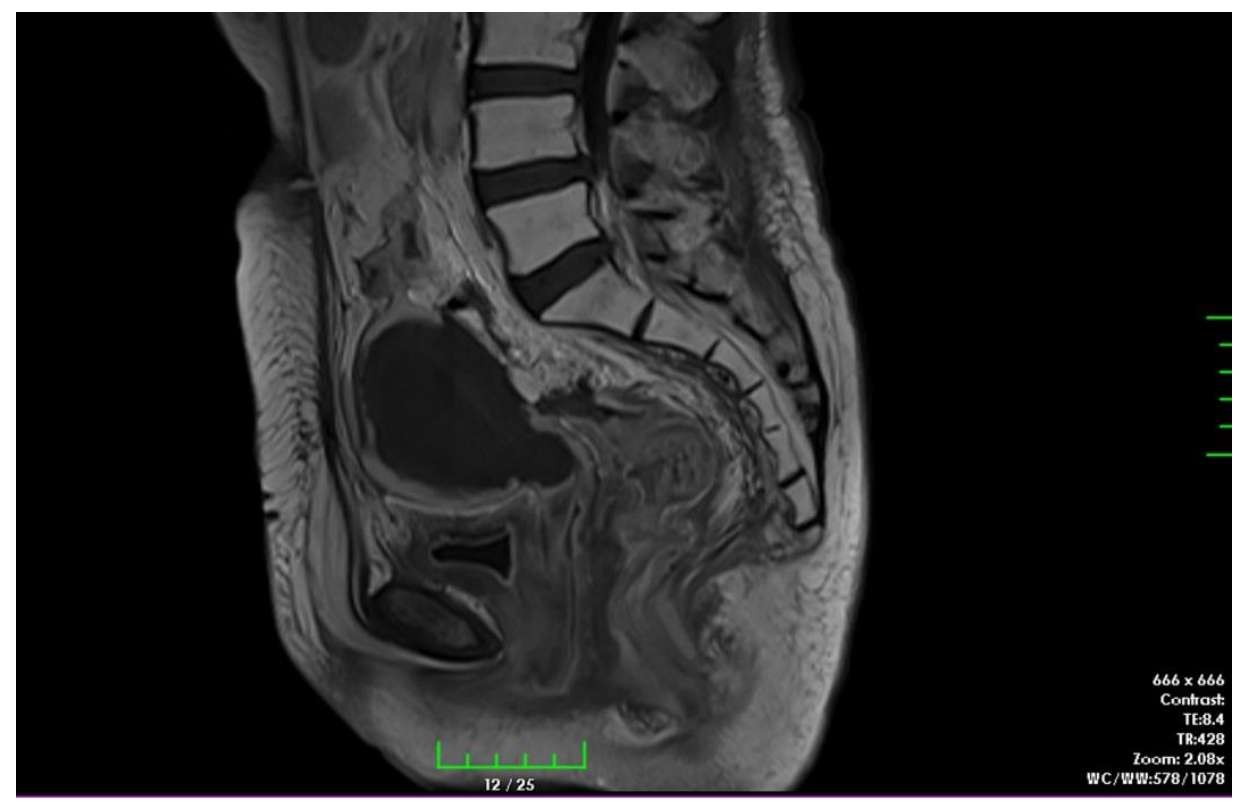

(b)

Figure 3: Pelvic MRI after a month of radiotherapy demonstrating complete response to the treatments (a) axial view, (b) sagittal view.

\section{Discussion}

Although there have been important advances in the management of women with cervical cancer, the optimal treatment for patients with metastatic disease to the non-regional lymph nodes at the time of diagnosis is still problematic, and there are relatively few randomized trials to guide treatment decisions. For women presenting with disease limited to the nodes (para-aortic and/or supraclavicular nodes), the prognosis is poor. Systemic chemotherapy, radiotherapy \pm chemotherapy may be reasonable depending on the institutional practice and patient preference [11]. For inguinal lymph node metastasis, the proximity of the inguinal lymph node to the pelvic radiotherapy portal makes it possible to include this lymph node chain to the external radiotherapy field. We reported a case of cervical cancer patient with left inguinal lymph node metastasis who underwent neoadjuvant chemotherapy followed by definitive radiotherapy.

Hauspy and colleagues reported a case of FIGO stage IIA squamous cervical cancer who had left inguinal lymph node metastasis at the time of diagnosis [12]. They performed combination of radiotherapy and chemotherapy for their patient. In their case, both right and left inguinal lymph nodes were positive and they applied $45 \mathrm{~Gy}$ of external radiotherapy to both groins and pelvis. Additionally the patient received $40 \mathrm{mg} / \mathrm{m}^{2}$ weekly cisplatin for six weeks. Brachytherapy was applied after external radiotherapy to a dose of $20 \mathrm{~Gy}$ in one fraction which was given by pulsedose-rate at $0.7 \mathrm{~Gy} / \mathrm{h}$. They achieved a complete response after a dose of brachytherapy. Different from the case by Hauspy and colleagues, we preferred neoadjuvant chemotherapy for our patient. We applied 6 cycles of neoadjuvant chemotherapy the patient had good-partial response. After neoadjuvant chemotherapy, we prefer definitive radiotherapy consisting of external radiotherapy and brachytherapy. We applied external radiotherapy to paraaortic, pelvic and bilateral inguinal lymph nodes, since our patient paraaortic and inguinal lymph node metastases in addition to pelvic lymph node metastases. As in the case of Hauspy et al, we achieved a complete response after a month of radiotherapy. 
There is limited data regarding to role of neoadjuvant chemotherapy in patients with locally advanced cervical cancer. In meta-analyses, it was shown that neoadjuvant chemotherapy followed by radical hysterectomy improved overall survival when compared to radical hysterectomy alone and radiotherapy alone in patients with locally advanced cervical cancer [13, 14]. Moreover in a meta-analysis of data on patients with stage IB1 and IIA cervical cancer found that neoadjuvant chemotherapy may reduce the need for adjuvant radiotherapy by decreasing tumor size and metastases, but indicated no overall survival [15]. However in another study it was shown that response to neoadjuvant chemotherapy was a strong prognostic factor for overall survival [16]. The European Organization for Research and Treatment of Cancer (EORTC) has been conducting a randomized study (EORTC55994) comparing neoadjuvant chemotherapy followed by radical hysterectomy versus concurrent chemo-radiotherapy for stage IB2, IIA2, and IIB patients with cervical cancer. The National Comprehensive Cancer Network (NCCN) guidelines also recommend adjuvant radiotherapy or concurrent chemo-radiotherapy for intermediate/high-risk patients who defined by pathological findings after RH (category 2b) [10]. Additionally in some countries FIGO stage IIB patients are treated with neoadjuvant chemotherapy followed by radical hysterectomy. In a phase II study, Tanioka and colleagues evaluated the role of neoadjuvant and adjuvant chemotherapy in patient with stage IB2, IIA2, or IIB cervical cancer [15]. They found that 2 and 5-year overall survival rates were $94.1 \%$ and $88.2 \%$ respectively. Therefore the authors concluded as chemotherapy consisting of dose-dense cisplatin+paclitaxel before and after radical hysterectomy achieved a good long-term survival and were feasible for patients with locally advanced cervical cancer. For FIGO stage IVB cervical cancer patients NCCN guideline recommends cisplatin based chemotherapy and individualized radiotherapy [10].

By the light of these findings, we preferred neoadjuvant chemotherapy consisting of carboplatin and pactitaxel as an initial treatment. After 6 cycles of chemotherapy our patient had good-partial response. Due to the proximity of the metastatic lymph node to the radiotherapy portal we could cover all the disease sites in to the treatment field. There is no data regarding to role of concurrent cisplatin and radiotherapy after neoadjuvant chemotherapy in metastatic patients. Our patient developed grad-4 neutropenia after $4^{\text {th }}$ cycles of chemotherapy nevertheless we could complete 6 cycles of chemotherapy. Moreover the radiotherapy field size was much extended since it included paraortic and bilateral groin lymph nodes in addition to standard pelvic area. Therefore we thought that the treatment might have been very toxic if we had used concurrent cisplatin. It was shown that the unwanted treatment breaks during the radiation therapy of cervical cancer associated with a decrease in local control [18]. The loss of local control approximated 1\%/day for treatment prolongation >30 days, and an analysis of stage subgroups showed this effect was predominant in Stage III-IV. Therefore we did not prefer to use concurrent cisplatin. Nevertheless at the end of the treatment we could achieve complete response.

In conclusion neoadjuvant chemotherapy followed by definitive radiotherapy consisting of external radiotherapy+brachytherapy seems to be a reasonable option in cervical cancer patients with inguinal lymph node metastasis. However there is still lack of data about the cervical cancer patients with non-regional lymph node metastases. Therefore new studies should be designed about this patient population.

\section{Conflict of Interest}

The authors declare that they have no conflict of interest 


\section{References}

1. Siegel R, Ward E, Brawley O, Jemal A. Cancer statistics, 2011: the impact of eliminating socioeconomic and racial disparities on premature cancer deaths. CA Cancer J Clin 61 (2011): 212.

2. Pecorelli S. Revised FIGO staging for carcinoma of the vulva, cervix, and endometrium. Int J Gynaecol Obstet 105 (2009): 103-104.

3. Ouldamer L, Marret H, Acker O, et al. Unusual localizations of sentinel lymph nodes in early stage cervical cancer: a review. Surg Oncol 21 (2012): e153-157.

4. Benedetti-Panici P, Maneschi F, Scambia G, et al. Lymphatic spread of cervical cancer: an anatomical and pathological study based on 225 radical hysterectomies with systematic pelvic and aortic lymphadenectomy. Gynecol Oncol 62 (1996): 19-24.

5. Ho CM, Chien TY, Huang SH, et al. Multivariate analysis of the prognostic factors and outcomes in early cervical cancer patients undergoing radical hysterectomy. Gynecol Oncol 93 (2004): 458-464.

6. Zhang HY, Sheng XG, Zhong Y, et al. The rule of metastatic pelvic lymph node distribution in patients with early stage cervical carcinoma. Zhonghua Zhong Liu Za Zhi. 30 (2008): 452-425.

7. Metcalf KS, Johnson N, Calvert S, et al. Site specific lymph node metastasis in carcinoma of the cervix: Is there a sentinel node? Int J Gynecol Cancer 10 (2000): 411.

8. Levenback C, Coleman RL, Burke TW, et al. Lymphatic mapping and sentinel node identification in patients with cervix cancer undergoing radical hysterectomy and pelvic lymphadenectomy. J Clin Oncol 20 (2002): 688 .

9. Ouldamer L, Marret H, Acker O, et al. Unusual localizations of sentinel lymph nodes in early stage cervical cancer: a review. Surg Oncol 21 (2012): e153-157.

10. NCCN guidelins version 1.2016. Cervical Cancer (2016).

11. Jason D, Wright JD. Management of recurrent or metastatic cervical cancer (2017).

12. Hauspy J, Verkinderen L, De Pooter C, et al. Sentinel node metastasis in the groin detected by technetiumlabeled nannocolloid in a patient with cervical cancer. Gynecol Oncol 86 (2002): 358-360.

13. Rydzewska L, Tierney J, Vale CL, et al. Neoadjuvant chemotherapy plus surgery versus surgery for cervical cancer. Cochrane Database Syst Rev 12 (2012): CD007406.

14. Neoadjuvant Chemotherapy for Locally Advanced Cervical Cancer Meta-analysis Collaboration. Neoadjuvant chemotherapy for locally advanced cervical cancer: a systematic review and meta-analysis of individual patient data from 21 randomised trials. Eur J Cancer 39 (2003): 2470-2486.

15. Kim HS, Sardi JE, Katsumata N, et al. Efficacy of neoadjuvant chemotherapy in patients with FIGO stage IB1 to IIA cervical cancer: an international collaborative meta-analysis. Eur J Surg Oncol 39(2013): 115124.

16. Landoni F, Sartori E, Maggino T, et al. Is there a role for postoperative treatment in patients with stage Ib2IIb cervical cancer treated with neo-adjuvant chemotherapy and radical surgery? An Italian multicenter retrospective study. Gynecol Oncol 132 (2014): 611-617. 
17. Tanioka M, Yamaguchi S, Shimada M, et al. Cisplatin with dose-dense paclitaxel before and after radical hysterectomy for locally advanced cervical cancer: a prospective multicenter phase II trial with a dosefinding study. Med Oncol 34 (2017): 134.

18. Fyles A, Keane TJ, Barton M, et al. The effect of treatment duration in the local control of cervix cancer. Radiother Oncol 25 (1992): 273-279.

(C) This article is an open access article distributed under the terms and conditions of the
Creative Commons Attribution (CC-BY) license 4.0 